pubescence est bien plus longue et fournie, la forme de son prothorax est toute différente et ses élytres sont beaucoup plus rugueux.

Basses-Pyrénées : grotte d'Oxibar, près de Camou-Cihigue; un exemplaire mâle pris sur une stalactite le 10 août 1913, au cours d'une exploration faite avec mon ami Ch. FAgniez.

Dans la même grotte se trouve l'A. Jeanneli Ab. C'était bien la dixième fois que je visitais la grotte d'Oxibar et c'est après huit heures de recherches dans cette petite grotte que j'ai trouvé ce nouvel Aphaenops. Ses affinités avec les $A$. Chaudoiri, A. Pandellei et $A$. Ehlersi me font penser que l'A. vasconicus est comme eux un habitant des grands bancs d'argile et que sa présence sur les stalactites d'Oxibar était accidentelle. Il est probable qu'il doit vivre dans des galeries argileuses inconnues, sous-jacentes à la grotte stalagmitée et qu'une cause inconnue l'avait attiré hors de son habitat normal. C'est vraisemblablement pour une raison analogue que l'A. Minos Lind. découvert en 1863 dans la grotte de Fontanet, près d'Ussat (Ariège) n'y a plus jamais été trouvé depuis.

Aphaenops orionis Fagniez(Bull. Soc.ent. Fr. [1913], p. 381). J'ai recueilli deux exemplaires de cette espèce nouvelle dans la grotte des sources de la Nive de Béhérobie, près de St-Jean-Pied-de-Port (Basses-Pyrénées). Ces exemplaires ne diffèrent du type que par leur taille qui est de $6, \breve{5}$ mill. (au lieu de 6,1 mill.). A. orionis semble donc bien être spécial aux grottes du bassin de la Nive, tandis qu'A. Jeanneli A b . peuple le bassin du gave de Mauléon.

\title{
Description d'un Pelorurus Mars. [CoL. Histeridae] nouveau
} par H. Desbordes.

Pelorurus Crampeli, n. sp. - Ovalis, convexus, niger, elytris viridi-coeruleis, tarsis brunneis. Frons parum concava, dupliciter punctulata, stria laterali. Pronotum lateribus fortiter punctatum, sed non ad basim, excepta punctorum linea ante scutellum; stria marginali lateribus et antice integra. Elytrorum striae profundae, subhumeralibus fere integris, 1-3 geminatis, 4-6 simplicibus, 4 integra, 5 dimidiata apicali, 6 integra; $2^{\circ}$ interstitio a medio ad basim valde dilatato. Pro- 
pygidium perspicue alutaceum, biimpressum, omnino punctatum, punctishaud densatis. Pygidium lateribus punctulatum, sed supra fere laeve. Prosternum punctulatum, striis lateribus integris. Mesosterni stria integra crenulata. Tibiis spinosis. - Long. วั mill.

Hab. : Fort-Crampel (Congo français). - Deux exemplaires m'ont été cédés par M. Le Moult, dont l'un est au Muséum de Paris, et l'autre dans ma collection.

Ovale, médiocrement convexe, noir, sauf lesélytres qui sont d'un beau bleu tirant sur le vert, et les tarses brunâtres. Front marqué d'une ponctuation double, légèrement concave. Pronotum fortement ponctué sur les côtés, la ponctuation s'affaiblissant à mesure qu'elle s'éloigne des côtés, et laissant lisse un espace à peu près triangulaire, égal au tiers environ de sa surface totale; une ligne de points tout contre la base, sur les deux tiers de celle-ci, bien alignés, sauf sur un petit espace de chaque còté de l'écusson. Stries èlytrales 1-3 profondes, formées chacune de deux stries entières, géminées à la base seulement, l'intervalle entre la 2 et la $3^{\text {e }}$ fortement élargi entre le milieu et la base; stries 4 - 6 simples, $4^{\mathrm{e}}$ entière, avec un retour en crochet à peine indiqué à la base, ว̌ apicale, n'atteignant pas tout à fait le milieu, avec un point à la base dans son prolongement; suturale entière, s'élargissant un peu au milieu et se rapprochant sensiblement de la suture en arrière. Propygidium marqué, sur un fond alutacé, d'une ponctuation médiocre et fort espacée. Pygidium médiocrement ponctué sur les deux faces inférieures seulement, à peu près lisse sur la face supérieure. Pièces sternales très finement pointillées; stries prosternales sinuées, entières en arrière, s'arrètant en avant à la striole transversale qui sépare la carène du bord antérieur; strie mésosternale entière, crénulée. Tous les tibias armés d'une rangėe d'épines de plus en plus fortes vers le sommel.

Par son système de striation élytrale, cette espèce se sépare de toutes celles du genre déjà connues, sauf de P. bruchoides M ars. Elle se distingue de cette dernière par les caractères suivants :

- Élytres franchement noirs. Front à ponctuation simple, à peine visible. Ligne de points de la base du pronotum ne s'étendant que sur une très petite partie de la base, et nulle en face de l'écusson. Ponctuation du propygidium nulle au milieu. כّe strie élytrale à peine marquée, s'arrêtant bien plus loin de l'apex que les autres. P. bruchoides Mars. 
- Élytres franchement bleus. Front à ponctuation double, bien marquée. Ligne de points de la base du pronotum ininterrompue, seulement irrégulière dans la région scutellaire. Ponctuation du propygidium également répandue sur toute la surface. 弓̈e strie élytrale bien marquée, presque aussi rapprochée de l'apex que les autres. P. Crampeli Desbordes.

\section{Cérambycides nouveaux de Colombie, appartenant au Musée} de Hambourg [COL.] $-1^{\text {re }}$ note

par É. Gounelle.

Les insectes dont nous donnons ici la description ont été recueillis à la fin de l'année 1912 par $\mathbf{M}$. W. Fritsche sur la montagne de La Garita (Colombie).

Ommata (Rhopalessa) Fritschei, n. sp. - o . Angusta, subglabra, testaceo-rufa, thorace saturiore, capite, elytrorum apice, clava femorum posticorum, tibiarum posticarum dimidio apicali, tarsis posticis necnon antennis nigris, articulis vero $7-11$ basi anguste flavo-annulatis; caput antice sensim attenuatum, rostro subbrevi, oculorum lobis inferioribus fere contiguis in fronte, vertice punctato; antennae dimidium corporis multo superantes, scapo parce punctulato, art. $\%$-10 obconicis et sensim incrassatis, intus perparum serratis, opacis; thorax latitudine longior, subcylindraceus, antice sensim paululum attenuatus, nitidus, punctis minutis inordinatim conspersus; scutellum semiovale, subtiliter flavido-pubescens; elytra thorace paululum latiora, abdominis segmentum quartum haud superantia, apice singulatim obliquiter sinuoseque truncata, a basi ad medium circiter sensim attenuata, dein subparallela et aeque angusta, dorso plana, punctis inordinatis cribrata, marginibus externis subabrupte declivibus carinulaque obtusa a disco longitudinaliter separatis, marginibus suturalibus paululum sensim dehiscentibus; femora modice clavata, postica basi pedunculata, elongata, abdominis autem segmentum quartum haud transeuntia, sparsim aspere punctata; tarsi postici subelongati, art. $1^{\circ} 2^{\circ}$ et $3^{\circ}$ simul sumptis paululum longiore; abdomen cylindraceum nitidum, segmentis 1-4 subaequalibus; corpus subtus parce fulvo-pilosum. - Long. 8,8 mill. - 2 exemplaires. 


\section{$2 \mathrm{BHL}$ Biodiversity Heritage Library}

1914. "Description d'un Pelorurus Mars. nouveau." Bulletin de la Société entomologique de France 1913, 384-386.

https://doi.org/10.5962/bhl.part.16305.

View This Item Online: https://www.biodiversitylibrary.org/item/36377

DOI: https://doi.org/10.5962/bhl.part.16305

Permalink: https://www.biodiversitylibrary.org/partpdf/16305

\section{Holding Institution}

Smithsonian Libraries

\section{Sponsored by}

Smithsonian

\section{Copyright \& Reuse}

Copyright Status: NOT_IN_COPYRIGHT

This document was created from content at the Biodiversity Heritage Library, the world's largest open access digital library for biodiversity literature and archives. Visit BHL at https://www.biodiversitylibrary.org. 